\title{
Innovative Technique for HPA Characteristics Extraction and Accurate Predistorsion Function Modeling
}

\author{
Blaise Mulliez \\ LAAS-CNRS, Université de Toulouse, \\ Toulouse INP-ENSEEIHT \\ Toulouse, France \\ E-mail: bmulliez@enseeiht.fr
}

\author{
Emmanuel Moutaye \\ LAAS-CNRS, Université de Toulouse, \\ Toulouse INP-ENSEEIHT \\ Toulouse, France \\ E-mail:emoutaye@enseeiht.fr
}

\author{
Hélène Tap \\ LAAS-CNRS, Université de Toulouse, \\ Toulouse INP-ENSEEIHT \\ Toulouse, France \\ E-mail: helene.tap@enseeiht.fr
}

\begin{abstract}
In telecommunications, the combination of modulations with non-constant envelope together with nonlinearities leads to strong distortions of the transmitted signals. The linearization purpose is to assure an optimal link transmission quality. In this article, an innovative method to extract linearization characteristics is presented. This technique, using directly experimental data rather than a High Power Amplifier (HPA) modeling, is fast and allows an important linearization accuracy and computational workload improvement compared to classical modeling techniques. It is then applied to determine the ideal linearizer AM/AM and AM/PM transfer characteristics to exploit three real HPA to their fullest.
\end{abstract}

Keywords - High Power Amplifier, predistorsion, characteristics extraction, function modeling

\section{I.INTRODUCTION}

With currently more than 300 active satellites in geostationary orbit dedicated to them, telecommunications represent the first spatial application. In this specific domain, power and spectral efficiencies are the two main concerns. In particular, some High Power Amplifiers (HPA), named Travelling Wave Tubes Amplifiers (TWTA), have to operate close to saturation so as to achieve the maximum power efficiency onboard the satellite. Nevertheless, this leads to amplitude and phase non-linearities. The main consequences are the link transmission quality deterioration and the spectrum pollution due to intermodulation products [1]. In other spatial applications, such as telemetry or payload data transfer, another kind of HPA, named Solid State Power Amplifiers (SSPA), are used, especially for low orbits. These amplifiers have to be highly linear in order to transfer correctly data from satellite to Earth without distortion. Nevertheless, depending on the waveform of the signal to transmit and the operating conditions of the payload, distortion may also appear.

There are several solutions to operate a HPA close to its saturation point without generating non-linearities. A first solution is to use post-distortion modules or equalization devices on Earth to compensate intermodulation products and intersymbol interferences at the receiver end [2]. However, this solution is complex, especially because the signal collected by the terminal undergoes strong modifications between the transmitter and the receiver, and is therefore hard to process. Another solution, called the predistortion method, consists in implementing a module among the equipment located before the HPA, to obtain a linear transfer characteristic of the whole transmission chain [3]-[5]. It is highly interesting for satellites with regenerative payloads in which the signal is available in baseband, before transposition. Indeed in this context, assuming that the frequency transposition does not distort the signal envelope, it is possible to apply a predistortion on the baseband signal before frequency transposition and HPA amplification. Particularly, amplitude and phase distortions issues can be processed independently.

Nevertheless, the definition of the predistortion characteristic as a function of the amplifier and time is not simple. Most often, modeling the HPA transfer distortion in amplitude and in phase as a function of the operating conditions is needed. If high resolution is required, the modeling process becomes heavy in time and processing resources. Likewise, if computational resources are low, resolution losses appear in the model. The modeling process of the HPA is not simple either. Eventually, finding the predistortion system optimal parameters as a function of the HPA and its drifts in time is complex, in particular if the linearization process has to be fast and precise.

Thus, the methods classically used to determine the predistorter transfer function require heavy computational workload, are not highly accurate and not always adapted to all the technologies used to develop HPA [6]-[8]. This paper presents a novel procedure to extract the predistorter characteristics from experimental data in the context of a single-carrier $\mathrm{AM} / \mathrm{AM}$ and $\mathrm{AM} / \mathrm{PM}$ characteristics linearization. In section II, the main two methods currently used to extract predistortion characteristics, as well as their drawbacks, are described. The innovative extraction technique is then detailed in section III; it is fast, accurate and requires very few computational resources. In section IV, this very interesting procedure is used to determine the predistortion functions for a $170 \mathrm{~W}$ Ka-band TWT amplifier, a $110 \mathrm{~W} \mathrm{Ku}$ band TWT amplifier and a 65W C and X-band SSP amplifier, whose characteristics have been provided by the French Space Agency (CNES). Finally, the conclusions are drawn in section V.

\section{PREDISTORSION CHARACTERISTICS DETERMINATION}

To determine predistortion and/or HPA transfer characteristics, several techniques are found in the literature, such as look-up tables (LUT) [10-12], or various mathematical 


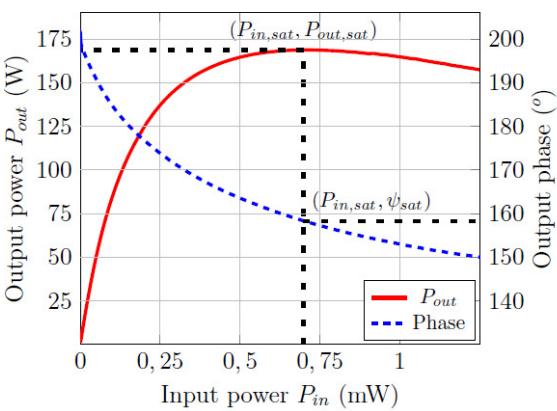

(a) $170 \mathrm{~W}$ Ka-band TWTA

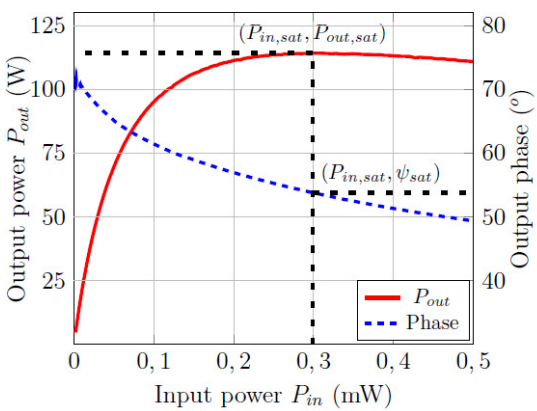

(b) $110 \mathrm{~W}$ Ku-band TWTA

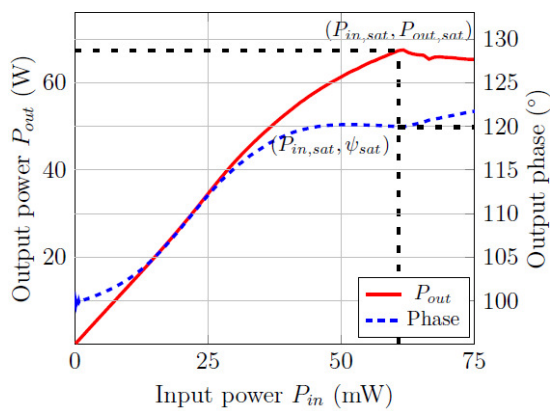

(c) $65 \mathrm{~W} \mathrm{C}$ and X-band SSPA

Fig. 1. AM/AM (plain line) and AM/PM (dotted line) characteristics at 20.2GHz of three HPA:

(a) 170W Ka-band TWTA, (b) 110W Ku-band TWTA, (c) 65W C and X-band SSPA (data provided by the CNES

models (Saleh [13], polynomial with or without memory [14], [15], Volterra [16], Hammerstein and Wiener [17], [18], Neural Networks [19]).

Many properties may justify the use of one of these techniques rather than the others and compromises have to be made. Mainly, a trade-off between precision and complexity of the model has to be determined. For instance, the extraction of the Saleh model parameters is almost immediate, whereas the Volterra series model requires a heavy computational workload. However, the Saleh model does not fit accurately to any $\mathrm{AM} / \mathrm{AM}$ and $\mathrm{AM} / \mathrm{PM}$ characteristics, whereas Volterra series can. Moreover, the HPAs may show memory effects [20]-[23]. This property may be defined as the dependence of the distortion on the input signal frequency variation. The ability to take into account these effects improves significantly the model accuracy. Eventually, some models can work equally with TWTA or SSPA, and are therefore highly generic. Nevertheless, in general, modeling methods are very application-specific. Table I summarizes the different models with their advantages and drawbacks.

\section{INNOVATIVE PREDISTORSION CHARACTERISTICS EXTRACTION TECHNIQUE}

To alleviate the aforementioned drawbacks, an innovative transfer characteristics extraction technique is developed [10]. It is usable to linearize single-carrier AM/AM and AM/PM characteristics of HPA such as the ones shown in Fig. 1. They represent the average power (plain line) and the phase (dotted line) of the signal at the amplifier output as a function of the average power of the signal injected at its input. The saturation point is also highlighted in this figure.

The extraction technique detailed below is fast, perfectly accurate and uses few resources as it only requires data tables. It follows 3 steps whose order is fixed, detailed in Fig. 2. The order of those steps is essential. Let's assume that the amplitude and phase characteristics of the linearizer are modeled by the functions $\mathrm{f}$ and $\varphi$ respectively and the amplitude and phase characteristics of the amplifier by the functions $\mathrm{g}$ and $\psi$ respectively, as shown in Fig. 3. This formalism has no physical nor mathematical reality but is only used for a demonstration purpose.

The average power at the HPA output is given by:

$$
P_{\text {out }, H P A}=g\left(P_{\text {in }, H P A}\right)
$$

The average power at the linearizer output is:

$$
P_{\text {in }, H P A}=f\left(P_{\text {in }, \text { Lin }}\right)
$$

Therefore:

$$
P_{\text {out }, H P A}=g \circ f\left(P_{\text {in }, \text { in }}\right)
$$

Calling $G_{\text {ideal }}$ the function modeling the ideal gain desired at the output of the system "Linearizer+HPA":

$$
G_{\text {ideal }}=P_{\text {out }, H P A} / P_{\text {in }, \text { Lin }}
$$

Then:

$$
(g \circ f)=G_{\text {ideal }} \cdot I_{D}
$$

with $I_{D}$ the identity function.

The linearizer AM/AM transfer function is therefore:

$$
f=g^{-1} \cdot G_{\text {ideal }}
$$

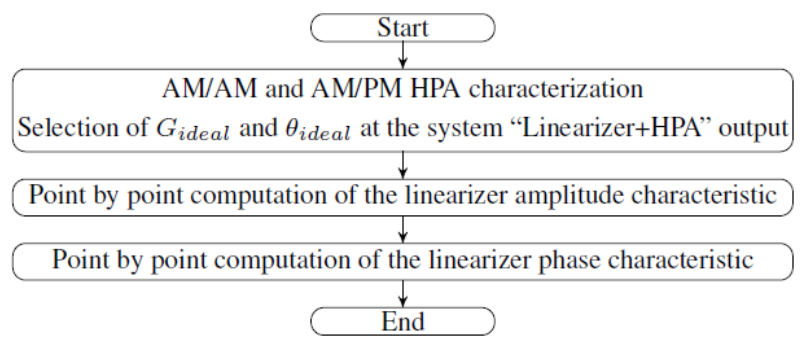

Fig. 2. Predistortion characteristics extraction steps

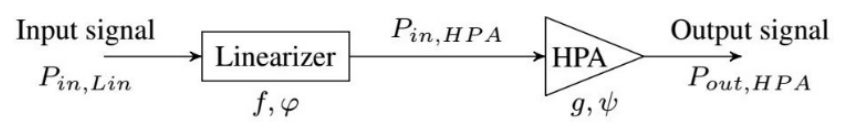

Fig. 3. "Linearizer+HPA" system and associated transfer function 
TABLE I. COMPARISON BETWEEN DIFFERENT HPA MODELING TECHNIQUES

\begin{tabular}{clccc}
\hline \hline Model & HPA & Memory effects & $\begin{array}{c}\text { Computational } \\
\text { workload }\end{array}$ & Model accuracy \\
\hline LUT & TWTA/SSPA & No & Simple & Low \\
Saleh & TWTA/SSPA & No & Simple & Low \\
Polynomial without memory & TWTA/SSPA & No & Simple & Low \\
Polynomial with memory & TWTA/SSPA & Yes & Complex & High \\
Volterra series & TWTA/SSPA & Yes & Very complex & Very high \\
Hammerstein & TWTA & Yes & Complex & High \\
Wiener & TWTA/SSPA & Yes & Complex & High \\
Hammerstein-Wiener & TWTA & Yes & Very complex & High \\
Parallel Wiener & TWTA/SSPA & Yes & Complex & High \\
Neural Netwok & TWTA/SSPA & Yes & Complex & Very high \\
This work & TWTA/SSPA & No & Simple & Very high \\
\hline \hline
\end{tabular}

TABLE II. HPA CHARACTERISTICS AND IDEAL OUTPUT VALUES NEEDED TO ESTABLISH THE MODEL

\begin{tabular}{ccccc}
\hline $\begin{array}{c}\text { HPA input } \\
\text { power }\end{array}$ & $\begin{array}{c}\text { HPA output } \\
\text { power }\end{array}$ & $\begin{array}{c}\text { HPA output } \\
\text { phase }\end{array}$ & Ideal gain & Ideal phase \\
\hline$P_{\text {in }, H P A}(i)$ & $P_{\text {out }, H P A}(i)$ & $\psi(i)$ & $G_{\text {ideal }}(i)$ & $\theta_{\text {ideal }}(i)$ \\
\hline$P_{\text {in }, H P A}(1)$ & $P_{\text {out }}, H P A(1)$ & $\psi(1)$ & $G_{\text {ideal }}(1)$ & $\theta_{\text {ideal }}(1)$ \\
$P_{\text {in }, H P A}(2)$ & $P_{\text {out }, H P A}(2)$ & $\psi(2)$ & $G_{\text {ideal }}(2)$ & $\theta_{\text {ideal }}(2)$ \\
$\vdots$ & $\vdots$ & $\vdots$ & $\vdots$ & $\vdots$ \\
$P_{\text {in }, H P A}(N)$ & $P_{\text {out }, H P A}(N)$ & $\psi(N)$ & $G_{\text {ideal }}(N)$ & $\theta_{\text {ideal }}(N)$ \\
\hline \hline
\end{tabular}

TABLE III. PREDISTORTION LINEARIZER TRANSFER AM/AM AND AM/PM CHARACTERISTICS CORRESPONDING TO THE VALUES MEASURED IN TABLE II

\begin{tabular}{cc|cc}
\hline \hline \multicolumn{2}{c|}{ AM/AM transfer function } & \multicolumn{2}{c}{ AM/PM transfer function } \\
\hline$P_{i n, L i n}(i)$ & $P_{i n, H P A}(i)$ & \multicolumn{1}{c}{$P_{i n, L i n}(i)$} & $\varphi(i)$ \\
\hline$P_{i n, L i n}(1)$ & $P_{i n, H P A}(1)$ & $P_{i n, L i n}(1)$ & $\varphi(1)$ \\
$P_{i n, L i n}(2)$ & $P_{i n, H P A}(2)$ & $P_{i n, L i n}(2)$ & $\varphi(2)$ \\
$\vdots$ & $\vdots$ & $\vdots$ & $\vdots$ \\
$P_{i n, L i n}(N)$ & $P_{i n, H P A}(N)$ & $P_{i n, L i n}(N)$ & $\varphi(N)$ \\
\hline \hline
\end{tabular}

So $f$ only depends on the desired gain characteristic and on the HPA properties. But, since the linearizer introduces a phase shift $\varphi\left(P_{i n, \text { Lin }}\right)$ and the HPA a phase-shift $\psi\left(P_{i n, H P A}\right)$, the total phase-shift at the HPA output is:

$$
\begin{aligned}
\theta_{\text {ideal }} & =\varphi\left(P_{\text {in }, \text { Lin }}\right)+\psi\left(P_{\text {in }, H P A}\right) \\
& =\varphi\left(P_{i n, L i n}\right)+\psi\left(f\left(P_{\text {in }, \text { Lin }}\right)\right)
\end{aligned}
$$

Therefore, the linearizer AM/PM transfer function is:

$$
\varphi=\theta_{\text {ideal }}-(\psi \circ f)
$$

$\varphi$ depends on the amplitude transfer characteristic. Consequently, the $\mathrm{AM} / \mathrm{AM}$ characteristics of the linearizer must be computed before the phase one. The same formalism is kept in the rest of the paper.

The first step of the extraction method is the HPA characterization: a continuous wave signal at a specific power $P_{\text {in.exp }}$ is injected in the HPA and the output power $P_{\text {out.exp }}$ and phase $\psi$ are extracted thanks to a vector network analyzer. By varying the input power on $\mathrm{N}$ values, the whole $\mathrm{AM} / \mathrm{AM}$ and $\mathrm{AM} / \mathrm{PM}$ characteristics are reported in a table (see the first three columns of Table II). The greater $\mathrm{N}$ is, the more precise the characteristics are. This table reflects the computational resources used in the extraction method and highlights how only few of these resources are needed.

Based on these data and on the gain and phase ideal functions desired at the HPA output only (respectively $G_{\text {ideal }}$ and $\left.\theta_{\text {ideal }}\right)$, which are summarized in table II, the linearizer characteristics can be constructed.

From Eq. 4 applied to the element $i$, the linearizer gain transfer function is directly determined by:

$$
P_{\text {in }, \text { in }}(i)=P_{\text {out }, H P A}(i) / G_{\text {ideal }}(i)
$$

The vectors couple $\left[P_{\text {in,Lin }}(i), P_{i n, H P A}(i)\right]$ represents the whole linearizer AM/AM characteristic.

Considering Eq. 7 applied to element $i$ with $\varphi(i)$ the phaseshift introduced by the linearizer excited at a power $P_{i n, \operatorname{Lin}}(i)$ and $\psi(i)$ the phase-shift introduced by the HPA excited at a power $P_{\text {in }, H P A}(i)$, it comes:

$$
\varphi(i)=\theta_{\text {ideal }}(i)-\psi(i)
$$




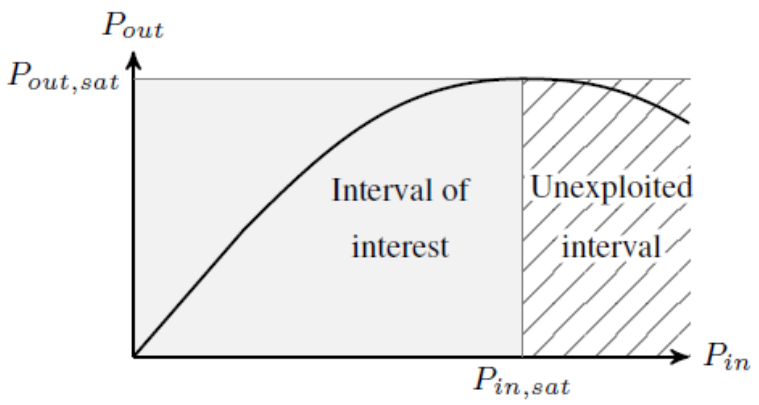

Fig. 4. Input power interval limitation

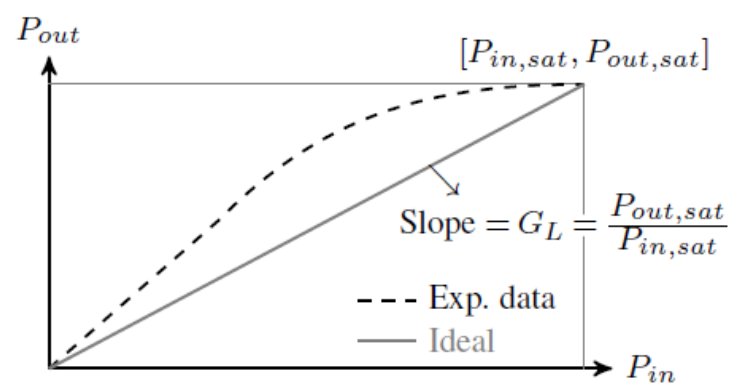

Fig. 5. Ideal AM/AM linearized transfer characteristic

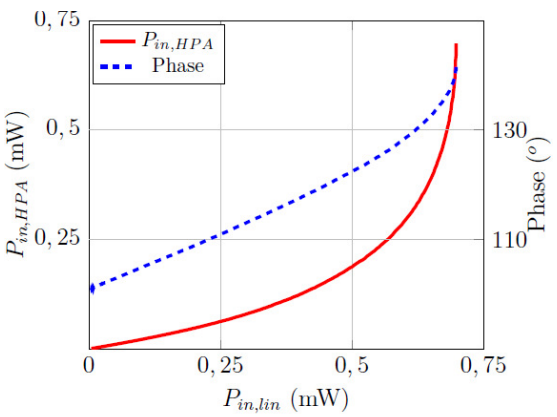

(a) $170 \mathrm{~W}$ Ka-band TWTA $\left(\theta_{L}=300^{\circ}\right)$

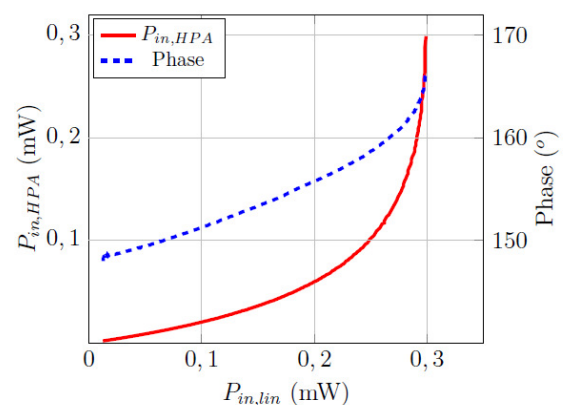

(b) $110 \mathrm{~W}$ Ku-band TWTA ) $\left(\theta_{L}=220^{\circ}\right)$

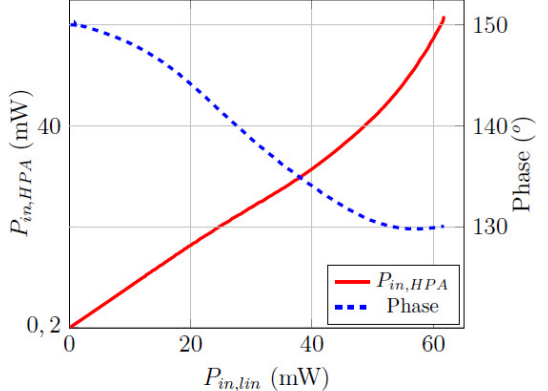

(c) $65 \mathrm{~W} \mathrm{C}$ and X-band $\operatorname{SSPA}\left(\theta_{L}=250^{\circ}\right)$

Fig. 6. Ideal linearizer AM/AM and AM/PM characteristics for the HPA presented in Fig. 1

The vectors couple $\left[P_{i n, L i n}(i), \varphi(i)\right]$ represents the whole linearizer AM/PM characteristic.

Through the 3 steps detailed above, and for each point reported in table II, two vectors couples shown in Table III and representing the linearizer transfer characteristics are extracted. This extraction technique is fast, requires no complex calculation and can be applied to any type of HPA, regardless of its technology, its nominal power or frequency, in order to linearize single-carrier AM/AM and AM/PM characteristics. Its accuracy is the highest possible since it requires no prior inexact modeling nor any approximation.

\section{APPLICATION TO THE LINEARIZATION OF A TWTA AND A SSPA}

In telecommunications, the combination of modulations with non-constant envelope together with nonlinearities leads to strong distortions of the transmitted signals. The linearization purpose is to assure an optimal link transmission quality. One linearization scheme in such a context is to select a fixed gain and a constant phase at the output of the system "Linearizer+HPA":

$$
\forall i \in[1 . . n], G_{i \text { deal }}(\mathrm{i})=G_{L} \text { and } \theta_{i \text { deal }}(i)=\theta_{L}
$$

It is not required that $\theta_{L}=0$. In practice, the value $\theta_{L}$ has to be chosen according to the linearization capabilities of the electronic circuit. On the opposite, for power efficiency purposes, the value of $G_{L}$ has to be carefully chosen. The following paragraph describes how to determine the best AM/AM linearized characteristic and the optimal $G_{L}$ value.

First, it is possible to restrain the input power interval of interest of the HPA AM/AM characteristic to [0,Pin,sat] as the
Fig. 4 shows. Indeed, as there is a bijection between [0, Pin,sat] and [0,Pout,sat], any output power can be reached with an input power belonging to $[0$, Pin,sat $]$. Then, the optimal linearized AM/AM characteristic is represented in Fig. 5. As a matter of fact, under those conditions, it is linear on the whole input power interval to $\left[0, P_{i n, s a t}\right]$. Moreover, since the saturation output power is reached, the HPA is exploited to its fullest and its efficiency is the highest possible.

The innovative technique described in the previous section is applied to the three characteristics shown in Fig. 1. The extracted predistorder transfer functions are plotted in Fig. 6.

\section{CONCLUSION}

In this paper, an innovative technique to compute predistorter $\mathrm{AM} / \mathrm{AM}$ and $\mathrm{AM} / \mathrm{PM}$ characteristics has been presented and applied to three real HPA whose experimental characteristics have been provided by the CNES. In the perspective of a potential generic linearization system design, this extraction technique is of high interest. As a matter of fact, it is fast, perfectly accurate, requires only few computational resources and can be used for any type of HPA, regardless of its technology, its nominal power or frequency and the desired linearized characteristics. Hence, the future development of an innovating fast and accurate single carrier predistortion module may considered the implementation of such a method. Even if the linearizer needs to be reconfigured due to HPA characteristics drifts (due to aging or temperature variations), the reconfiguration time and precision may be largely improved thanks to this method. Thus, this method could grant linearizer designers an important gain of time, performances and implementation efficiency. 


\section{ACKNOWLEDGMENTS}

This research was supported by the French National Centre for Space Studies (CNES) and the Fren French General Armament Direction (DGA). We are thankful to our colleagues Laurent Gatet and Geoffroy Soubercaze-Pun from CNES, who provided expertise that greatly assisted the research.

\section{REFERENCES}

[1] J.Sombrin, "Optimisation criteria for power amplifiers", Int. J. Microw. Wirel. T., 2011,3(1), pp. 35-45

[2] M. Ben Mabrouk, G. Ferré, E. Grivel, N. Deltimple, "A new baseband post-distortion technique for power amplifiers in OFDM-based cognitive radio systems”, IEEE ISCAS'15, May 2015, pp. 1486-1489

[3] G. Breed, "An Overview of Common Techniques for Power Amplifier Linearization”, High Frequency Electronics, Feb. 2010, pp. 44-46

[4] R. Marsalek, P. Jardin, G. Baudoin, "From post-distortion to predistortion for power amplifiers", IEEE Comm. Letters, 2003 , 7(7), pp. $308-310$

[5] B. Mulliez, E. Moutaye, H. Tap, L. Gatet, F. Gizard, "Predistortion system implementation based on analog neural networks for linearizing HPAs transfer characteristics", S2IS, 2014, 7(1), pp. 400-422

[6] V. Volterra, "Theory of Functionals and of Integral of IntegroDifferential Equations", Dover Publications, Inc, N.Y., 1958

[7] O. Hammi, S. Boumaiza, F.-M. Ghannouchi, "On the robustness of digital predistortion function synthesis and average power tracking for highly nonlinear power amplifiers", IEEE T. Microw. Theory, 2007, 55(6), pp. 1382-1389

[8] R. Sperlich, J. Sills, J. Kenney, "Closed loop digital predistortion with memory effects using genetic algorithms", IEEE MTT-S Int. Micr., 2005, pp. 1557-1560

[9] B. Mulliez, G. Soubercaze-Pun, L. Gatet, E. Moutaye, H. Tap, "Procédé de configuration d'un circuit corrigé comprenant un circuit imparfait et un circuit de prédistorsion", French Patent, 1453 773, 2013

[10] M. Jin, S. Kim, D. Ahn, D.-G. Oh, J.-M. Kim, “A fast LUT predistorter for power amplifier in OFDM systems", 14th IEEE Int. Sym. Pers. In.,Vol. 2, pp. 1894 - 1897

[11] B. Feuvrie, D. Magaye, Y. Wang, "Efficient Baseband Digital Predistorter Using Lut for Power Amplifier (PA) with Memory Effect",
American Journal of Electrical and Electronic Engineering, 2014, 2(3), pp. $72-81$

[12] H. Jiang, P. Wilford, "Digital predistortion for power amplifiers using separable functions", IEEE T. Signal Proces., Aug. 2010, 58(8), pp. $4121-4130$

[13] M.-M. Shammasi, S.-M. Safavi, "Performance of a predistorter based on Saleh model for OFDM systems in HPA nonlinearity", ICACT'12, 2012, pp. 148-152

[14] D. R. Morgan, Z. Ma, J. Kim, M. G. Zierdt, J. Pastalan, “A generalized memory polynomial model for digital predistortion of RF power amplifiers", IEEE T. Signal Proces., Oct. 2006, 54(10), pp. 3852-3860

[15] L. Ding, G.-T. Zhou, D.-R. Morgan, Z. Ma, J.-S. Kenney, J. Kim, C.-R. Giardina, "A robust digital baseband predistorter constructed using memory polynomials", IEEE T. Commun., Jan. 2004, 52(1), pp. 159165

[16] H. Rezgui, F. Rouissi, A. Ghazel, "Digital Predistorter Design Using a Reduced Volterra Model to Linearize GaN RF Power Amplifiers", Radioengineering, Sept. 2018, 27(3), pp. 909-916

[17] Y. Ding, H. Ohmori, A. Sano, "Adaptative predistortion for High power amplifier with linear dynamics”, IEEE MWSCAS'04, July 2004, pp. 121124

[18] T. Wang, J. Ilow, "Compensation of Nonlinear Distortions with memory Effects in Digital Transmitters", IEEE CNSR'04, May 2004, pp. 3-9

[19] N. Naskas, Y. Papananos, "Neural-network-based adaptive baseband predistortion method for RF power amplifiers", IEEE T. Circuits-II, Nov. 2004, 51(11), pp. 619-623

[20] J. Kenney, P. Fedorenko, "Identification of RF Power Amplifier Memory Effect Origins using Third-Order Intermodulation Distortion Amplitude and Phase Asymmetry", IEEE MTT-S Int. Micr., June 2006, pp. 1121-1124

[21] P. Roblin, D. Root, J. Verspecht, Y. Ko, J.-P. Teyssier, "New Trends for the Nonlinear Measurement and Modeling of High-Power RF Transistors and Amplifiers With Memory Effects", IEEE T. Microw Theory, June 2012, 60(6), pp. 1964,1978

[22] J. Vuolevi, T. Rahkonen, J.. Manninen, "Measurement technique for Characterizing Memory Effects in RF Power Amplifiers", RAWCON 2000, Sept 2000, pp. 195-198

[23] H. Ku, J. S. Kenney, "Behavioral modeling of nonlinear RF power amplifiers considering memory effects", IEEE T. Microw. Theory, Dec. 2003, 51(12), pp. 2495-2504 